\title{
The Effects of Psychological Stress on Plasma Interleukin-6 Activity in Rats
}

\author{
LIN G. LEMAY, ARTHUR J. VANDER AND MATTHEW J. KLUGER \\ Department of Physiology, The University of Michigan Medical School, Ann Arbor, MI 48109
}

Received 4 January 1990

\begin{abstract}
LEMAY, L. G., A. J. VANDER AND M. J. KLUGER. The effects of psychological stress on plasma interleukin-6 activity in rats. PHYSIOL BEHAV 47(5) 957-961, 1990. - The purpose of this study was to determine the effects of a particular psychological stress, exposure to an open-field, on plasma IL- 6 activity in rats. Plasma IL-6 activity was $40.6 \pm 7.2$ units $/ \mathrm{ml}$ in control rats, $105 \pm 6.8$ units $/ \mathrm{ml}$ after 30 minutes exposure to an open-field, and $221 \pm 17$ units $/ \mathrm{ml}$ after 60 minutes of exposure $(p=0.0003)$. There was a positive correlation $(\mathrm{r}=.71, p=0.043)$ between the change in plasma IL-6 activity and body temperature. However, we conclude, based on earlier data relating plasma IL-6 activity to body temperature changes following injection of lipopolysaccharide, that the plasma levels of IL- 6 following exposure to an open-field are not high enough to account for the rise in body temperature observed in rats during this stress. In conclusion, these experiments indicate that exposure to psychological stress can elevate the plasma concentration of IL-6, a known mediator of the acute phase response.
\end{abstract}

Stress hyperthermia Body temperature Cytokines Anesthesia Methoxyflurane Ketamine hydrochloride Xylazine

ONE response to certain psychological stresses, which is common both to animals and humans, is an elevation in body temperature termed "stress-induced hyperthermia" $(2-4,11,15,18)$. A portion of this hyperthermia can be blocked by cyclooxygenase inhibitors such as sodium salicylate and indomethacin $(4,11,18)$. These data support the hypothesis that part of the stress-induced hyperthermia is mediated by prostaglandins in the central nervous system (CNS) (20). It is currently thought that during fever, cytokines such as interleukin-1 (IL-1) and tumor necrosis factor (TNF) are responsible for the rise in central nervous system prostaglandins $(7,20)$. Recently, interleukin-6 (IL-6), a cytokine that is thought to be responsible for part of the acute phase response $(9,10,14,16,22)$, has also been found to be capable of producing fevers (10), and we have found that IL-6-induced fevers are blocked by indomethacin (12). This raises the possibility that stress-induced hyperthermia may be caused by increased release of IL-6.

The major hypothesis we have tested in this study is that exposure of rats to open-field stress results in an elevation in plasma levels of IL-6. We were also interested in whether the rise in body temperature and the observed increase of plasma IL-6 activity in response to open-field stress showed adaptation after multiple exposures to the open-field. Another hypothesis that was tested was that the stress of exposure to anesthetics would result in a rapid rise in plasma levels of IL-6.

METHOD

\section{Animals}

Forty-six specific pathogen-free male Sprague-Dawley rats weighing $180-220 \mathrm{~g}$ were obtained from Charles River (Portage, MI). Rats were housed at $23-25^{\circ} \mathrm{C}$ with a $12 / 12 \mathrm{hr}$ light-dark cycle and given ad lib tap water and rodent chow. Rats were individually housed for about 2 weeks before the experiment.

\section{Measurement of Body Temperature}

Core temperature was measured by biotelemetry using transmitters implanted intraperitoneally (IP) (Mini Mitter, Inc., Sunriver, OR) (17). The transmitters were implanted at least 4 days before experiments. Each transmitter was calibrated prior to implantation. Output (frequency in $\mathrm{Hz}$ ) was monitored by a mounted antenna placed under each rat's cage and fed into a peripheral processor (Dataquest III system, Mini Mitter, Inc.) connected to an IBM-PC. Temperatures were recorded at 1-minute intervals.

\section{IL-6 Bioassay}

IL- 6 activity in plasma was measured using the IL-6-dependent B-9 hybridoma cell line kindly provided by Dr. Lucien Aarden (Central Laboratory of the Netherlands) $(1,21)$. The B-9 cells were cultured in Iscove's modified Dulbecco's medium (IMDM; Life Technology, Inc.) supplemented with human recombinant IL-6 (8 units/ml obtained from L. Aarden), $20 \mu \mathrm{M}$ 2-mercaptoethanol, $5 \%$ heat-inactivated fetal calf serum (FCS), $100 \mathrm{IU} / \mathrm{ml}$ penicillin and $100 \mu \mathrm{g} / \mathrm{ml}$ streptomycin. Cells were washed once in the above medium without added IL- 6 before the addition of the plasma samples or known amounts of human rIL-6, also provided by Dr. Aarden.

To run the $\mathrm{IL}-6$ assay, $100 \mu \mathrm{l}$ of 1:10 diluted plasma sample was combined with 5000 B-9 cells in $100 \mu \mathrm{IMDM} / 5 \%$ FCS in flat-bottom microtiter plates (Corning) for a final volume of 200 $\mu l$. All samples were run in duplicate. The control medium, which 
contained no IL-6, was run in quadruplicate. In addition to the undiluted plasma samples, a serial dilution of each sample was assayed. Serial dilution was performed because our standard curve indicated that high levels of IL-6 result in an inhibition of growth of the B-9 cells. Each sample was serially diluted initially a maximum of 320-fold. Cells were pulsed at 68-72 hours with 0.5 $\mu \mathrm{Ci}{ }^{3} \mathrm{H}$ thymidine, harvested onto glass fiber filter strips (Cambridge Technology, Inc.), and the radioactivity incorporated into DNA was counted by a $\beta$-Scintillation Counter (Packard Instrument Company). For each assay, a standard curve was run with recombinant human IL-6. One unit of IL-6 is equal to the amount that caused half-maximum proliferation in the standard curve. From this standard curve, a best-fit regression was calculated for the rising portion of the curve in the linear range. The equation for this best fit line was used to calculate IL-6 activity in plasma samples.

For the serially diluted samples, all counts per minute (cpm) that fell within 2 standard deviations of the baseline cpm (i.e., within the $95 \%$ confidence interval) were excluded to reduce the potential error resulting when converting from cpm to units of IL-6 by multiplying by the dilution factor. The largest calculated IL- 6 activity that fell outside the $95 \%$ confidence intervals was taken as the IL- 6 value; this ensured that all data used fell in the steep linear portion of the standard curve.

To document that plasma IL-6-like activity in the rat plasma was really due to IL-6, we measured this activity in the B-9 assay after addition of rabbit antibody to mouse recombinant IL-6 (kindly supplied by Dr. Richard Nordan, National Cancer Institute). The B-9 assay was run as described above except that $2 \mu \mathrm{g}$ of antibody against mouse IL- 6 was added to the serially diluted plasma sample and incubated for 1 hour before addition of the B-9 cells.

\section{Stress Paradigm}

One method for inducing psychological stress in rats is exposure to an open-field $(11,18)$. The open-field used in these experiments consisted of a $60^{\prime \prime} \times 38^{\prime \prime} \times 81^{\prime \prime}$ high white acrylic spray finish temperature-controlled chamber (Warren Sherer) illuminated by two fluorescent lights suspended from above. The temperature within the open-field box $\left(25-26^{\circ} \mathrm{C}\right)$ was similar to that in the rat's home cages. The experimental protocol for the stress involved quickly removing the rat from his own small cage and placing him into the open-field. After the appropriate period of time, the rat was returned to his cage. Control animals were not placed in the open-field. In the adaptation study, the rats were exposed to the open-field for 30 minutes per day for the first seven days and for 60 minutes for the last three days. To minimize possible circadian variability, exposure to the open-field occurred only between 9 a.m. and 3 p.m.

\section{Plasma Samples}

In the open-field study, blood was withdrawn from the rats either prior to or following exposure to the open-field by cardiac puncture immediately after it was killed by cervical dislocation to avoid any potential acute stress-induced changes in IL- 6 as a result of anesthesia. Blood was collected into a heparinized syringe in rats that were removed from their home cages (controls) or from the open-field stress chamber and cervically dislocated within 20 seconds.

To determine whether anesthesia did in fact have an acute effect on plasma levels of IL-6, blood was withdrawn from the rats by cardiac puncture immediately after it was killed by cervical dislocation following no treatment (control group) or treatment

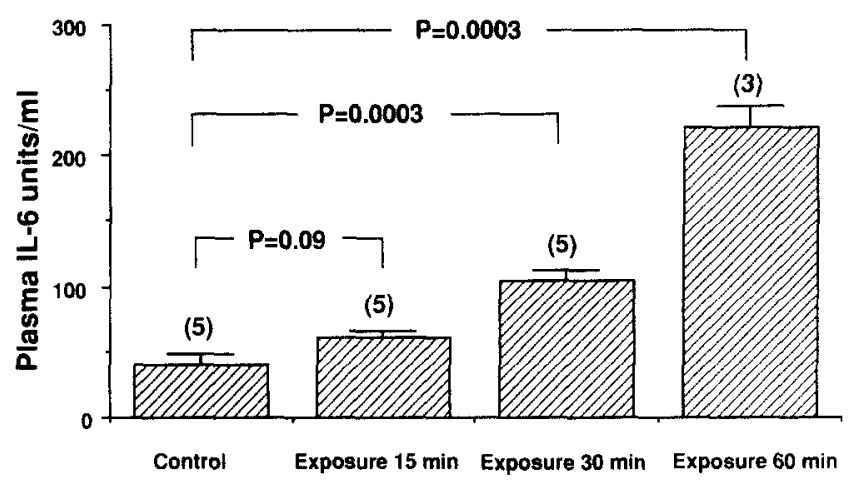

FIG. 1. Plasma IL-6 activity in control rats (not exposed to open-field), or rats exposed to open-field for 15,30 or 60 minutes. Sample size is indicated in parentheses. $p$ Values were calculated by comparing each of the open-field-treated groups to the control group using Student's $t$-tests corrected for multiple comparisons with the method of Bonferroni. Each bar represents a distinct group of rats. In other words, this was not a longitudinal study of a single group of rats in order to avoid any possible effect of repeated blood sampling on IL-6. Thus, we only sampled for IL-6 once from each rat.

with either a combination of $70 \mathrm{mg} / \mathrm{kg}$ ketamine hydrochloride (Ketaset, Bristol Laboratories) and $10 \mathrm{mg} / \mathrm{kg}$ xylazine (Rompun, Mobay Corporation) injected intramuscularly, or the gas anesthetic methoxyflurane (Pitman-Moore, Inc.). The blood was collected as soon as the rats became unconscious, which was about 1-2 minutes.

Plasma was separated by centrifugation of the freshly drawn blood and stored at $-20^{\circ} \mathrm{C}$ until assayed for IL-6.

\section{Data Analysis}

Data are presented as mean $\pm S$.E. Statistical differences were generally determined by analysis of variance followed either by Student's $t$-tests or paired $t$-tests corrected in both cases for multiple comparisons by the method of Bonferroni.

\section{RESULTS}

Exposure to an open-field resulted in increases in IL-6 activity proportional to the period of stress (Fig. 1). Plasma IL-6 activity in control rats was $40.6 \pm 7.2$ units $/ \mathrm{ml}$. After exposure to the open-field for 15 minutes, the plasma IL-6 activity was $60.3 \pm 5.3$ units $/ \mathrm{ml}(p=0.09)$. After 30 -minutes exposure, plasma IL-6 activity was $105 \pm 6.8$ units $/ \mathrm{ml}(p=0.0003)$ and after 60 minutes it was $221 \pm 17$ units $/ \mathrm{ml}(p=0.0003)$. We confirmed that the bioassay was specific for IL- 6 by demonstrating that $95 \%$ of the IL-6 activity in rats' plasma following open-field stress was neutralized by antibody against mouse IL-6.

The effect of repeated exposure to the open-field on plasma IL-6 activity is shown in Fig. 2. It should be noted that this was not a longitudinal study of a single group of rats, in order to avoid any possible effect of repeated sampling of blood on IL-6. Thus, we only sampled for IL-6 once from each rat. The plasma IL-6 in rats subjected to a 30-minutes open-field exposure for the first time was $105 \pm 6.8$ units $/ \mathrm{ml}$, as already shown in Fig. 1, which was not significantly different from the values for IL-6 in the 2nd or 4th exposure to the open-field. In the eighth and tenth exposures, we increased the duration of exposure to 60 minutes. The mean of plasma IL-6 in the 8th exposure was $161 \pm 55$ units $/ \mathrm{ml}$, and for the 10th exposure was $78.5 \pm 46$ units $/ \mathrm{ml}$ ( $p=0.14$; Students' $t$-test). Among the four rats exposed for the 10th time, the plasma IL-6 


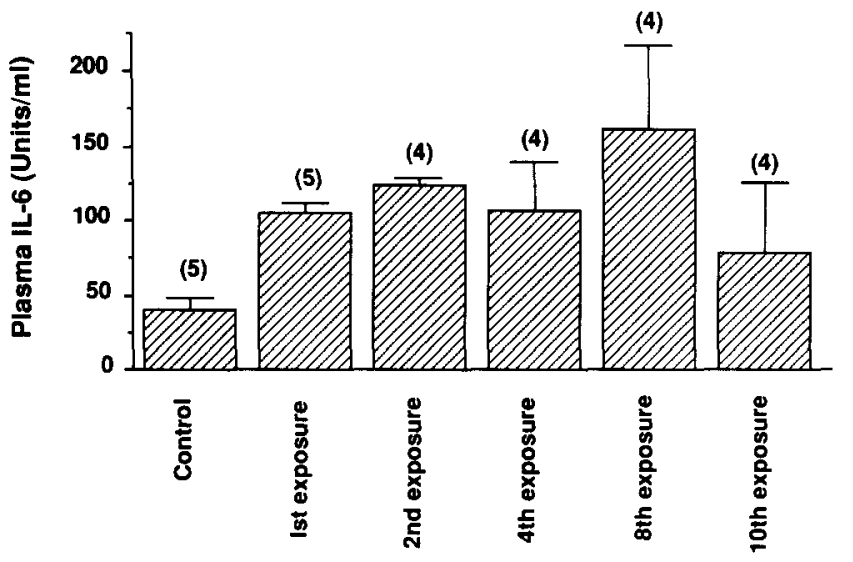

FIG. 2. Plasma IL-6 activity in control (not exposed to open-field) rats, or rats subjected to repeated multiple daily exposures to the open-field. For the first seven days, each rat was exposed to the open-field for 30 minutes each day. For days eight to ten, the exposure was for 60 minutes. The data for the "control" and "1st exposure" are the data of Fig. 1 for "control" and "30 min exposure." As in Fig. 1, each bar is a distinct group of rats.

activities were in the control range in three of these rats (mean $=$ $32.3 \pm 4.2$ units $/ \mathrm{ml}$ ), whereas one rat had a high IL-6 activity ( 217 units $/ \mathrm{ml}$ ). Based on the statistical criterion of "detection of extremes" (8), we redid the analysis after excluding this high IL-6 value. When this was done, the average value for the 10th exposure was now only approximately one-fifth that for the 8th exposure, with a borderline statistical significance of $p=0.053$; Students' $t$-test.

The body temperature changes in response to repeated exposure to the open-field are shown in Fig. 3A. The changes in body temperature were not significantly different among the first exposure to the ninth exposure. In Fig. $3 \mathrm{~A}$, the numbers of rats decreased with the days of exposure to the open-field due to the sacrificing of animals required for blood sampling. Figure 3B presents the body temperature changes in the group of rats that were exposed to the open-field for all 10 days. There was a significant decrease in the rise of body temperature between the 8th exposure to the open-field (first day of 60 -minutes stress) and the 10 th exposure ( 3 days of 60 -minutes stress) ( $p=0.023$; paired $t$-test).

In order to determine the relationship between the change in core temperature and plasma IL-6, we plotted the value for plasma IL-6 activity vs. the same rat's change in temperature as a result of exposure to the open-field (Fig. 4). The correlation coefficient relating the change in plasma IL-6 activity and body temperature, based on a polynomial regression, was $0.71(p=0.043)$. Note that one rat, which was exposed to the open-field at the 10th time (solid squares), showed both high $\mathrm{IL}-6$ and high temperature. The changes in temperature and plasma IL-6 activities in the other three rats exposed to the open-field the 10th time had both reduced plasma IL-6 values and reduced change in body temperature.

Anesthesia had no statistically significant acute effect on plasma IL-6 activity $(p=0.35$ ) (Fig. 5).

\section{DISCUSSION}

Plasma IL-6 activity increased significantly during exposure of rats to an open-field. In another study from this laboratory, it has been shown that stress-induced rises in body temperature of rats are not the result of increases in general physical activity (13), and thus we conclude that psychological stress itself (rather than a
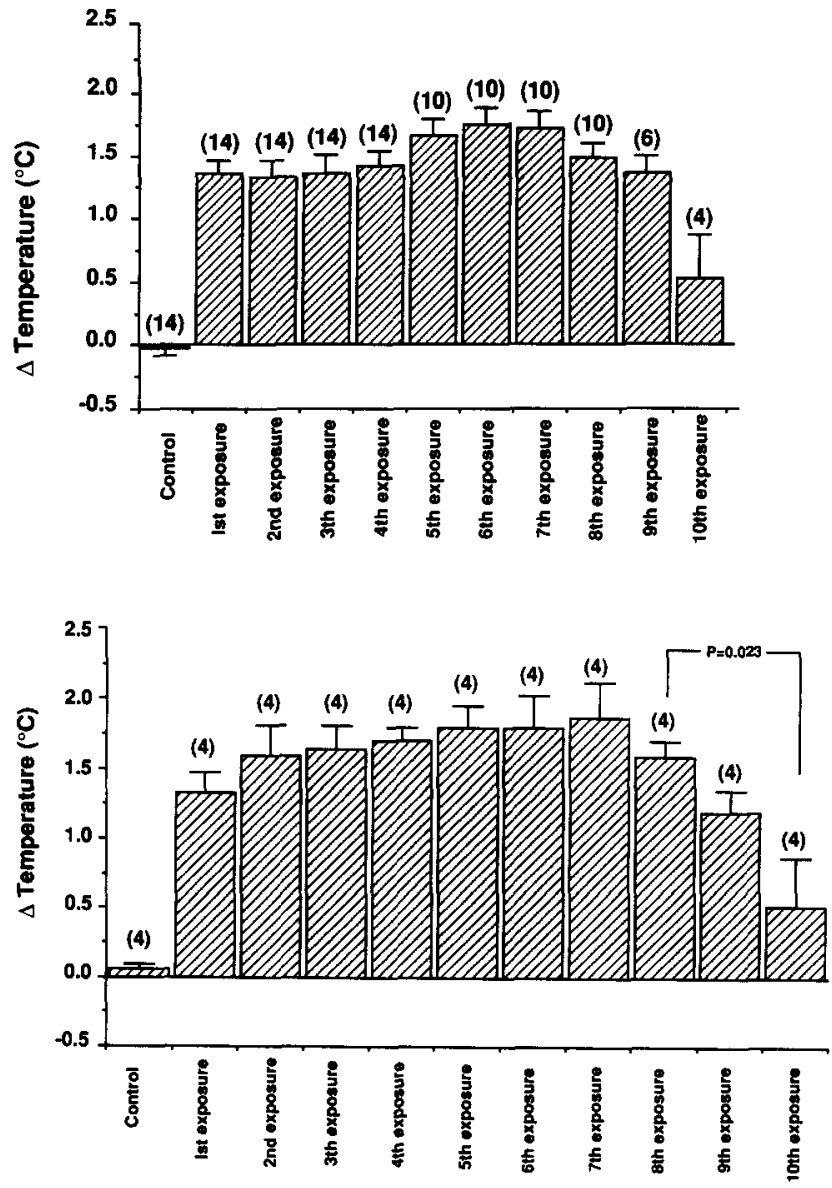

FIG. 3. (A) Change in core temperature of control rat and rats exposed to an open-field for 30 minutes per day for the first seven days and for 60 minutes for the last three days. Twelve of the rats in this study are the same rats shown in Fig. 2 (4th exposure, 8th exposure and 10th exposure). Sample size is indicated in parentheses. The average temperatures of the rats prior to the start of the experiment on day one was $37.2^{\circ} \mathrm{C}$. (B) Change in core temperature of rats before and after rats were exposed to an open-field for 30 minutes per day for the first seven days and for 60 minutes for the last three days. These data are the subset of those plotted in (A) who experienced a full 10 days of exposure to the open-field. Sample size is indicated in parentheses. $p=$ level of statistical significance.

combination of psychological and physical stress) can raise the plasma concentration of IL-6.

Our results also indicate that repeated exposures to open-field results in adaptation in both body temperature and plasma IL-6 secretion. Because we had observed no tendency for adaptation after 7 days of exposure to the open-field, we increased the stress period in order to see whether adaptation of temperature changes or plasma IL-6 can be produced by the combination of multiple exposure and longer period exposure. Therefore, because of the change in protocol on day 8, we do not know whether the adaptation was due mainly to the extra days of exposure (days $8-10)$ or to the more prolonged exposures ( 60 minutes) beginning on day 8.

There was a statistically significant correlation between plasma IL-6 and the change in core temperature induced by the stress (Fig. 4). However, despite this correlation, we do not believe that the plasma concentrations achieved are high enough to account for the stress-induced rises in body temperature observed. In a previous 


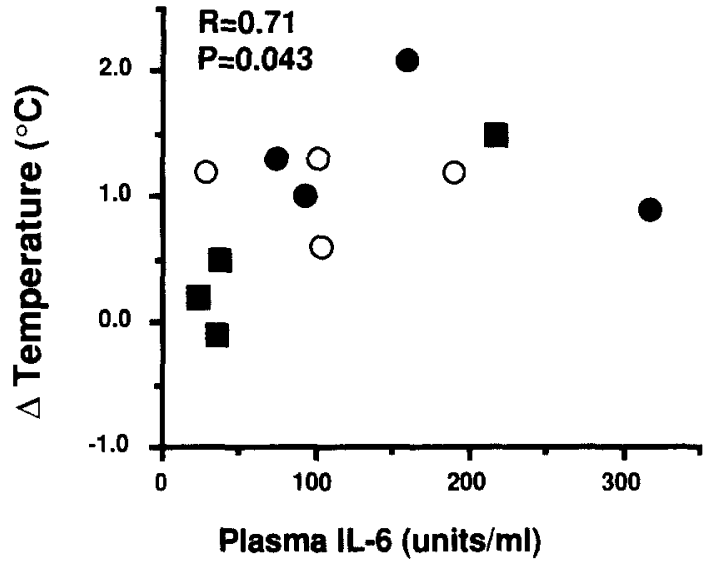

FIG. 4. Plasma IL-6 activity vs. change in body temperature for individual rats after exposure to open-field. The data used are from individual rats exposed to the open-field the fourth, eighth and tenth times (based on data shown in Figs. 2 and 3A). Each point is the value for one rat at the relevant time. Open circles represent the 4th exposure to open-field, closed circles the 8 th exposure, and squares the 10 th exposure. $\mathrm{R}=$ correlation coefficient and $p=$ level of statistical significance.

study, in which we measured the plasma IL-6 activity and temperature in rats at one and two hours following intraperitoneal injection with $1 \times 10^{6}$ units of recombinant human IL-6, we found that plasma IL- 6 activity rose to $266 \pm 105$ units/ml at one hour and to $193 \pm 32$ units $/ \mathrm{ml}$ at two hours (12), values similar to those seen in the present stress experiments; these plasma concentrations of IL-6 did not result in a significant rise in body temperature. This analysis, however, applies only to plasma IL-6; it is possible that stress hyperthermia is triggered by local release of IL-6 into the brain (19) or other organ, and that the increase in plasma IL-6 simply reflects leakage of this cytokine into the blood.

The study on the effects of anesthesia on changes in plasma

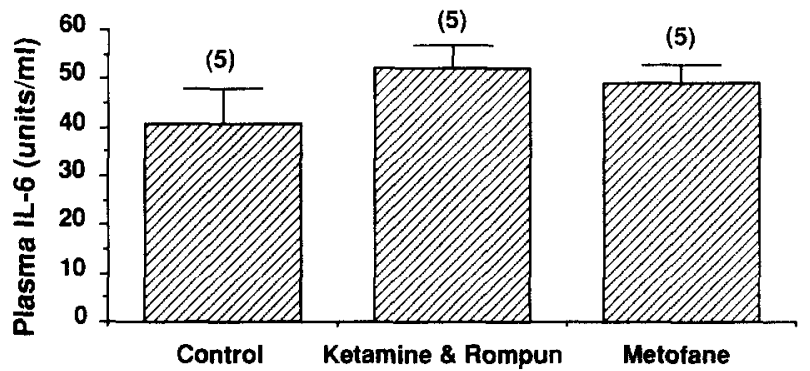

FIG. 5. Plasma IL-6 activity in control (no treatment) rats, or rats treated with either the combination of $70 \mathrm{mg} / \mathrm{kg}$ ketamine hydrochloride and 10 $\mathrm{mg} / \mathrm{kg}$ xylazine injected intramuscularly, or the gas anesthetic methoxyflurane. The control data are the same data from Fig. 1. Sample size is indicated in parentheses.

IL-6 activity provided us with the information that the method of collecting blood from rats for assay for IL-6 by injection of the anesthetics ketamine hydrochloride and xylazine, or inhalation of the anesthetic methoxyflurane, caused no greater IL-6 increase than did cervical dislocation.

The finding that psychological stress can increase the plasma concentration of IL-6 is, to our knowledge, the first demonstration that nonphysical stimuli can increase secretion of a cytokine thought to be important in both specific and nonspecific immunity. Other studies $(5,6)$ have shown that exercise is associated with an increase in IL-1, but it is possible that the stimulus in exercise is physical trauma within the exercising muscle. The pathways by which psychological stress increases IL-6 secretion remain to be determined.

\section{ACKNOWLEDGEMENTS}

We thank Dr. Lucien A. Aarden for generously providing us with the B-9 cell line and human recombinant IL-6 and Dr. Richard Nordan for providing us with antibody against mouse IL-6. Research supported in part by NIH DK 38984 and NIH NS 23633.

\section{REFERENCES}

1. Aarden, L. A.; de Groot, E. R.; Schaap, O. L.; Lansdorp, P. M. Production of hybridoma growth factors by human monocytes. Eur. J. Immunol. 17:1411-1416; 1987.

2. Blasig, J.; Hollt, V.; Bauerle, U.; Herz, A. Involvement of endorphins in emotional hyperthermia of rats. Life Sci. 23:2525-2532; 1978.

3. Briese, E.; De Quijada, M. G. Colonic temperature of rats during handing. Acta Physiol. Latinoam. 20:97-102; 1970.

4. Briese, E.; Cabanac, M. Emotional fever and salicylate. Proc. of 28 th Intern. Cong. Physiol. Sci., vol. 32 in Advances in physiological sciences. Pergamon Press-Akademiai Kiado; 1980:161-163.

5. Cannon, J. G.; Dinarello, C. A. Increased plasma interleukin-1 activity in women after ovulation. Science $227: 1247-1249 ; 1985$.

6. Cannon, J. G.; Kluger, M. J. Endogenous pyrogen activity in human plasma after exercise. Science 220:617-619; 1983.

7. Dinarello, C. A.; Cannon, J. G.; Wolff, S. M.; Bernheim, H. A.; Beutler, B. A.; Cerami, A.; Figari, I. S.; Palladino, M. A.; O'Connor, J. V. Tumor necrosis factor (cachectin) is an endogenous pyrogen and induces production of interleukin 1. J. Exp. Med. 163:1433-1450; 1986.

8. Dixon, W. J.; Massey, F. J., Jr. Introduction to statistical analysis. New York: McGraw-Hill; 1983:377-379.

9. Gauldie, J.; Richards, C.; Harnish, D.; Baumann, H. Interferon $\beta 2$ is identical to monocytic HSF and regulates the full acute phase protein response in liver cells. In: Powanda, M. C.; Oppenheim, J. J.; Kluger,
M. J.; Dinarello, C. A., eds. Progress in leukocyte biology, vol. 8 . Monokines and other non-lymphocytic cytokines. New York: Alan R. Liss Inc.; 1988:15-20.

10. Helle, M.; Brakenhoff, J. P. J.; Groot E. R. D.; Aarden, L. A. Interleukin 6 is involved in interleukin 1-induced activities. Eur. J. Immunol. 18:957; 1988.

11. Kluger, M. J.; O'Reilly, B.; Shope, T. R.; Vander, A. J. Further evidence that stress hyperthermia is a fever. Physiol. Behav. 39: 763-766; 1987.

12. LeMay, L. G.; Vander, A. J.; Kluger, M. J. The role of IL-6 in fever in the rat. Am. J. Physiol. 258:R798-R803; 1990.

13. Long, N. C.; Vander, A. J.; Kluger, M. J. Antiserum against tumor necrosis factor (TNF) increases stress hyperthermia in rats. Am. J. Physiol. 258:R332-R337; 1990.

14. Nijsten, M. W. N.; de Groot, E. R.; ten Duis, H. J.; Klasen, H. J.; Hack, C. E.; Aarden, L. A. Serum levels of interleukin- 6 and acute phase response. Lancet Oct 17:921; 1987.

15. Renbourn, E. T. Body temperature and pulse rate in boys and young men prior to sporting contests. A study of emotional hyperthermia: with a review of the literature. J. Psychosom. Res. $4: 149-175 ; 1960$.

16. Santhanam, U.; Tatter, S. B.; Helfgott, D. C.; Ray, A.; Ghrayeb, J.; May, L. T.; Sehgal, P. B. Structure, genetics and function of human " $\beta 2$-interferon/B-cell stimulatory factor-2/hepatocyte stimulating factor' (Interleukin-6). In: Powanda, M. C.; Oppenheim, J. J.; Kluger, M. J.; Dinarello, C. A., eds. Progress in leukocyte biology, vol. 8. 
Monokines and other non-lymphocytic cytokines. New York: Alan R. Liss Inc.; 1988:8:29-34.

17. Scales, W. E.; Kluger, M. J. Effect of antipyretic drugs on circadian thythm in body temperature of rats. Am. J. Physiol. 253:R306-313; 1987.

18. Singer, R.; Harker, C. T.; Vander, A. J.; Kluger, M. J. Hyperthermia induced by open-field stress is blocked by salicylate. Physiol. Behav. $36: 1179-1182 ; 1986$.

19. Spangelo, B. L.; Login, I. S.; Judd, A. M.; Isakson, P. C.; MacLeod,
R. M. Release of interleukin-6 from rat hypothalamus. Endocrinology; in press.

20. Stitt, J. T. Prostaglandin E as the neural mediator of the febrile response. Yale J. Biol. Med. 59:137-149; 1986.

21. Van Oers, M. H. J.; van der Heyden, A. A. P. A. M.; Aarden, L. A. Interleukin-6 (IL-6) in serum and urine of renal transplant recipients. Clin. Exp. Immunol. 71:312-318; 1988.

22. Wong, G. C.; Clark, S. C. Multiple actions of interleukin-6 with a cytokine network. Immunol. Today 9:137-139; 1988. 